\title{
Eosinophilic Endomyocarditis: Rare Cases with Uncertain Prognosis
}

Thoene L*, Stahnke VC, Lotz J and Unterberg-Buchwald C

Department of Radiology, University Hospital Göttingen, Germany

\section{Case 1}

A 67-year-old patient presented in a rural hospital with an increasing fatigue and worsening of her general state of health. She had a known rheumatoid arthritis that was diagnosed in 1990. One year before she suffered from a first cerebral ischemia that recurred in the following months resulting in a sensomotoric hemiparesis. In addition she underwent Hemicolectomy with chemotherapy two months before the actual admission. The laboratory showed a leukocytosis, an eosinophilia and an increase in C-reactive protein. A transthoracic Echocardiogram (Figure 1) showed two large thrombi in both ventricles and a moderately reduced function of both ventricles (visual $\mathrm{EF}=35-40 \%$ ). Earlier coronary angiography did not show any pathology that could explain the reduced ventricular function. The rural hospital started a therapy with oral steroids (prednisone 60 $\mathrm{mg}$ ) and transferred the patient to the university hospital for further diagnosis. Cardiac MRI showed a highly reduced left ventricular ejection fraction $(\mathrm{EF}=27 \%)$ and a normal right ventricular ejection fraction $(\mathrm{EF}=55 \%)$ with huge thrombi in both ventricles (Figure 2). Late Gadolinium Enhancement of the entire endocardium and partial patchy late enhancement of the myocardium was shown (Figure 3). Due to the large thrombi myocardial biopsy was contraindicated. The most appropriate diagnosis of Loeffler's endocarditis was deduced from the synopsis of the above descripted findings. As a hypereosinophilic syndrome can be associated with a FIP1L1-PDGFRA mutation this was ruled out. Besides a standard heart failure therapy (ACE, diuretics and beta blocker) anticoagulation with low molecular heparin was started and converted to a therapy with dabigatran during the inpatient stay.

Six weeks later left ventricular function improved to $35 \%$, however the size of the biventricular thrombi decreased only minimally. Oral anticoagulation was changed to warfarin but the thrombus size did not reduce under therapeutic anticoagulation and EF kept constantly reduced. Due to the comorbidities, the age and the general very poor clinical status the patient preferred a palliative treatment and care at home.

\section{Case 2}

A 64-year-old patient presented with progressive dyspnea, cough and hemoptysis in a rural hospital. The diagnostic results including an Eosinophilia (11.9\%) led to the suspicion of Churg-Strauss-syndrome. Treatment with steroids improved the patient's state of health. Due to an acute renal failure the patient was transferred to our hospital.

The patient was dialyzed and a biopsy of the kidney showed a focal segmental glomerulosclerosis without any clues for vasculitis. A pulmonary biopsy showed chronic inflammation but no Eosinophilic granulocytes and no signs of vasculitis. Echocardiography showed a global hypokinesia, left ventricular ejection fraction was $46 \%$, earlier coronary angiography had not shown any vascular pathology. A FIP1L1-PDGFRA mutation was ruled out. High dose steroid therapy was induced with reduction every week from $80 \mathrm{mg}$ to $20 \mathrm{~mm}$.

Six weeks later the patient still had dyspnea. Echocardiography now revealed a thrombus in the left ventricle and patchy Endomyocardial Late Gadolinium Enhancement (Figure 4). Oral anticoagulation with

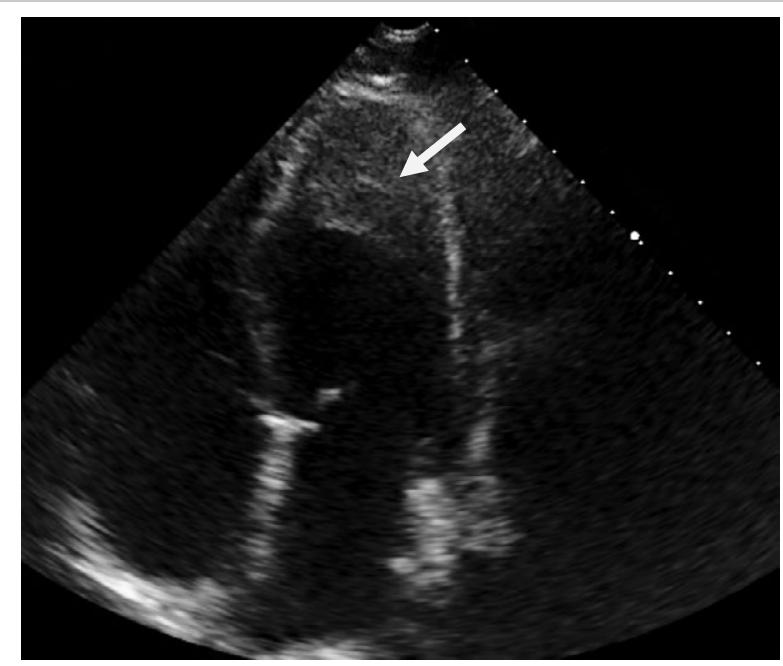

Figure 1: Case 1: Transthoracic echocardiography showing the large thrombus in the left ventricle.

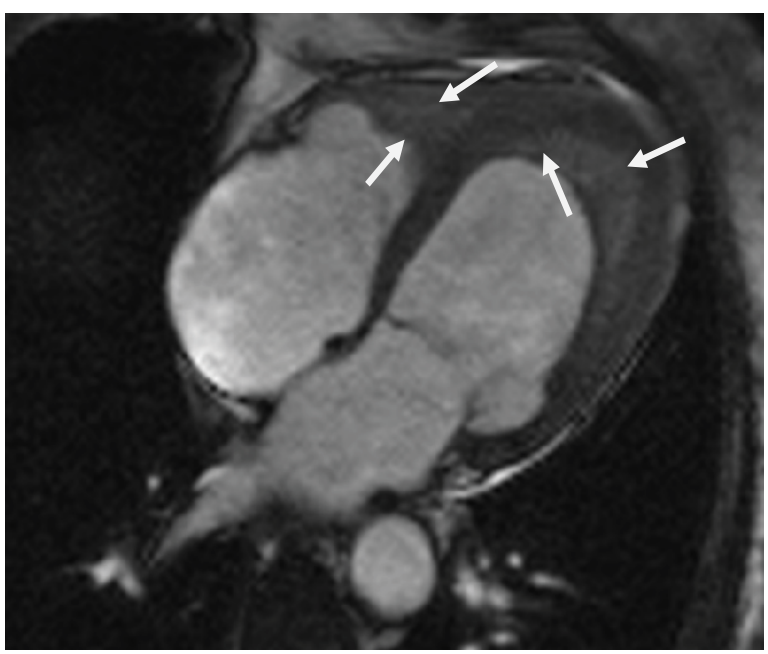

Figure 2: Case 1: True fast imaging with steady-state free precession, four chamber view, with biventricular thrombi.

*Corresponding author: Thoene L, Department of Radiology, University Hospital Göttingen, Robert-Koch-Straße 40, 37075 Goettingen, Germany, Tel: 0049-5513966066; Fax: 0049-551-399606; E-mail: lotte.thoene@med.uni-goettingen.de

Received January 18, 2016; Accepted January 28, 2016; Published February 01, 2016

Citation: Thoene L, Stahnke VC, Lotz J, Unterberg-Buchwald C (2016) Eosinophilic Endomyocarditis: Rare Cases with Uncertain Prognosis. Cardiovasc Pharm Open Access 5: 171. doi:10.4172/2329-6607.1000171

Copyright: ( $) 2016$ Thoene L, et al. This is an open-access article distributed under the terms of the Creative Commons Attribution License, which permits unrestricted use, distribution, and reproduction in any medium, provided the original author and source are credited. 
Citation: Thoene L, Stahnke VC, Lotz J, Unterberg-Buchwald C (2016) Eosinophilic Endomyocarditis: Rare Cases with Uncertain Prognosis. Cardiovasc Pharm Open Access 5: 171. doi:10.4172/2329-6607.1000171

Page 2 of 3

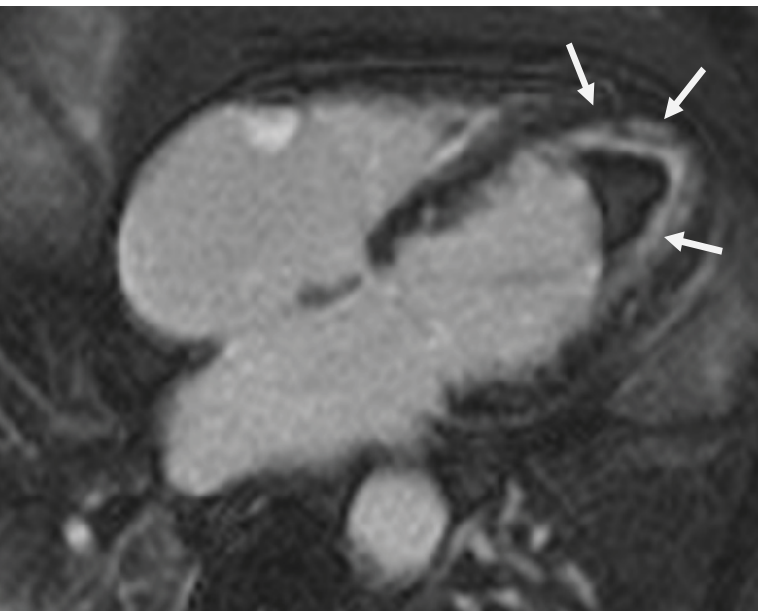

Figure 3: Case 1: Phase-sensitive inversion recovery (PSIR) single-shot TrueFISP, four chamber view, showing distinct Endomyocardial Late Gadolinium Enhancement of the left and right ventricular myocardium.

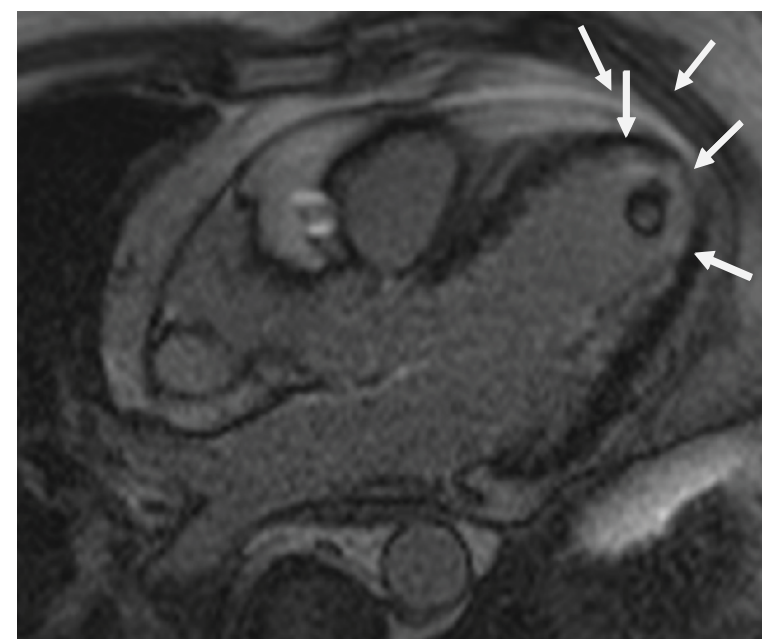

Figure 4: Case 2: Phase-sensitive inversion recovery (PSIR) single-shot TrueFISP, left ventricular outflow tract view, showing the Endomyocardial Late Gadolinium Enhancement and the ventricular thrombus.

warfarin was started and steroid therapy was planned to be reduced from $20 \mathrm{mg}$ to $7.5 \mathrm{mg}$. However, three months later the patient presented with increasing dyspnea and a worsening of his general state of health accompanied by a recurrence of the eosinophilia (13.1\%). Another cardiac MRI was performed and showed a reduced left ventricular ejection fraction (EF 25\%) with persisting patchy Endocardial Late Gadolinium Enhancement, the thrombus had vanished. Myocardial biopsy showed an elapsed Endomyocarditis. In summary the diagnosis of Eosinophilic endocarditis resulted in a therapy with cortisone pulse combined with cyclophosphamide. The patient improved initially: EF increased to $50 \%$ and dyspnoe disappeared. However, a few months later all symptoms recurred. CT showed a progress of the pulmonary manifestation. Thus the cyclophosphamide therapy was stopped and a therapy with rituximab was started, which led to a significant and quick improvement of all symptoms. Therapy was changed to an immunosuppressive therapy with azathioprine and prednisolone 20 mg. Both clinical symptoms and CT findings improved during the following weeks and the patient is in clinically good conditions until today.

\section{Discussion}

In the given cases both patients presented with dyspnea, depressed cardiac function and an eosinophilia. Eosinophilic endo-/myocarditis is a rare subtype of myocarditis that can lead to focal or diffuse Myocardial Infiltration [1,2]. The symptoms of the patients with Eosinophilic Myocarditis may vary and be unspecific [3,4]. Our first patient presented with an eosinophilia and unexplained heart failure leading to the suspicion of an Eosinophilic Myocarditis, coronary artery disease or diastolic heart failure was excluded previously. However, eosinophilia is too unspecific to prove the diagnosis as some patients with EM do not have elevated eosinophils and others with eosinophilia do not suffer from EM [4,5]. In this patient MRI showed severe diffuse left ventricle dysfunction and ventricular thrombi. The pattern of Late Gadolinium Enhancement (LGE) with subendocardial LGE in nearly all segments combined with patchy intramyocardial areas is typical for Loeffler's endocarditis [6,7] and was the decisive factor for the suspected diagnosis as it was not possible to take a myocardial biopsy due to the large non resolving thrombi. Though, a secured diagnosis of a Loeffler's endocarditis can only be made by taking a myocardial biopsy which is the diagnostical gold standard [8]. Nevertheless cardiac MRI has been proved to be a reliable and sensitive diagnostic method [9]. Possible differential diagnoses of eosinophilia and reduced cardiac function are Loeffler's endocarditis, Churg-Strauss syndrome, early giant cell myocarditis, hypersensitivity reactions (medication induced), parasitic infection, tropical Endomyocardial fibrosis or malignancy $[3,10]$. The patient was treated under the suspected diagnosis with steroids and standard therapy of heart failure. Eosinophilic Myocarditis is a rare clinical entity and so far no standard treatment has been established. The most important treatment goal is to ensure hemodynamic stability [3]. There are various case reports that document a successful therapy with corticosteroids [1-4]. In the case of our first patient after several weeks of anticoagulation and steroid-therapy, the biventricular thrombi were not significantly reduced in size. Ventricular function remained markedly reduced but the patient chose to stop further immunosuppressive therapy. Former studies monitoring venous thrombi showed a wide individual variation with respect to resolution of the thrombi showed persisting thrombi respectively incomplete resolution of the thrombi over the period of 6 month in several cases [11]. With respect to the resolution of arterial thrombi the data is very limited but it can be assumed that the resolution should be taking about the same amount of time. Thus it does not obligatory have to be a prolonged resolution of the biventricular thrombi but can be interpreted as a normal variation. Another explanation could be the late initiation of the therapy as our patient did already have very large thrombi. However, it remains unclear why our patient did not respond to the steroid therapy as the MRI showed persistent late gadolinium enhancement and the leukocytes remained elevated. A FIP1L1PDGFRA mutation was ruled out in both cases. In some patients hypereosinophilic syndrome can be associated with this mutation and patients show a poor respond to steroid therapy [12].

In the second case finally myocardial biopsy proved the suspected diagnosis of Eosinophilic Endo- Myocarditis due to Churg-Strausssyndrome in combination with the very typical findings in cardiac MRI. There were no focal or diffuse scars that could be explained by a coronary heart disease or other structural disease nor were they typical for other forms of myocarditis.

The second patient was treated by a combined immunosuppressive therapy which led to a clinical improvement of his symptoms. Ventricular function improved into near normal and thrombus 
Citation: Thoene L, Stahnke VC, Lotz J, Unterberg-Buchwald C (2016) Eosinophilic Endomyocarditis: Rare Cases with Uncertain Prognosis. Cardiovasc Pharm Open Access 5: 171. doi:10.4172/2329-6607.1000171

resolved under anticoagulation.

In both cases MRI has proved to be a reliable method to diagnose Eosinophilic Endomyocarditis and gave important information during the follow-up.

\section{References}

1. Kawano S, Kato J, Kawano N, Yoshimura Y, Masuyama H, et al. (2011) Clinical features and outcomes of eosinophilic myocarditis patients treated with prednisolone at a single institution over a 27-year period. Intern Med 50: 975-981.

2. Thambidorai SK, Korlakunta HL, Arouni AJ, Hunter WJ, Holmberg MJ (2009) Acute eosinophilic myocarditis mimicking myocardial infarction. Tex Heart Inst J 36: 355-357.

3. Rizkallah J, Desautels A, Malik A, Zieroth S, Jassal D, et al. (2013) Eosinophilic myocarditis: two case reports and review of the literature. BMC Res Notes 6: 538.

4. Al Ali AM, Straatman LP, Allard MF, Ignaszewski AP (2006) Eosinophilic myocarditis: case series and review of literature. Can J Cardiol 22: 1233-1237.

5. Weller PF, Bubley GJ (1994) The idiopathic hypereosinophilic syndrome. Blood 83: $2759-2779$
6. Greulich S, Ferreira VM, Dall'Armellina E, Mahrholdt H (2015) Myocardial Inflammation-Are We There Yet? Curr Cardiovasc Imaging Rep 8: 6.

7. Cummings KW, Bhalla S, Javidan-Nejad C, Bierhals AJ, Gutierrez FR, et al (2009) A pattern-based approach to assessment of delayed enhancement in nonischemic cardiomyopathy at MR imaging. Radiographics 29: 89-103.

8. Caforio AL, Pankuweit S, Arbustini E, Basso C, Gimeno-Blanes J, et al. (2013) Current state of knowledge on aetiology, diagnosis, management, and therapy of myocarditis: a position statement of the European Society of Cardiology Working Group on Myocardial and Pericardial Diseases. Eur Heart J 34: 26362648

9. Takahashi N, Murakami Y, Shimada T, Kashima Y, Nakamura K, et al. (2001) Detection of eosinophilic myocarditis using contrast-enhanced magnetic resonance imaging: case report. Can Assoc Radiol J 52: 20-22.

10. Ogbogu PU, Rosing DR, Horne MK 3rd (2007) Cardiovascular manifestations of hypereosinophilic syndromes. Immunol Allergy Clin North Am 27: 457-475.

11. Nijkeuter M, Hovens MM, Davidson BL, Huisman MV (2006) Resolution of thromboemboli in patients with acute pulmonary embolism: a systematic review. Chest 129: 192-197.

12. Cools J, DeAngelo DJ, Gotlib J, Stover EH, Legare RD et al. (2003) A tyrosine kinase created by fusion of the PDGFRA and FIP1L1 genes as a therapeutic target of imatinib in idiopathic hypereosinophilic syndrome. N Engl J Med 348: 1201-1214. 